\title{
LOEWY LENGTH OF PERFECT RINGS
}

\author{
B. L. OSOFSKY 1
}

ABSTRACT. We construct a family of left and right perfect rings whose left and right Loewy lengths are arbitrary preassigned infinite ordinals.

Let $R$ be a left perfect ring with Jacobson radical $N$. Bass [2] defines a well-ordered chain of ideals $\left\{N_{\alpha}\right\}$ of $R$ by $N_{0}=(0), N_{\alpha}$ $=$ the inverse image of the right socle of $R / N_{\beta}$ for $\alpha=\beta+1$, and $N_{\alpha}$ $=\mathrm{U}_{\beta<\alpha} N_{\beta}$ for $\alpha$ a limit ordinal. Since $R$ is left perfect, there exists a least ordinal $\alpha_{0}$ such that $N_{\alpha_{0}}=R . \alpha_{0}$ is called the right Loewy length of $R$. If $R$ is right perfect, a symmetrical definition of the left Loewy length of $R$ holds. In the case that $R$ is semiprimary, this is precisely the traditional definition (see for example [1, p. 104]), and the left and right Loewy lengths are equal (they are both equal to the index of nilpotency of $N$ ).

Bass asks if there are any restrictions on the right Loewy length of a left perfect ring $R$. Since $R_{R}$ is finitely generated, this length cannot be a limit ordinal. We show that this is the only restriction. Indeed, let $\alpha$ and $\beta$ be any pair of infinite ordinals. Then there exists a ring $R$ which is right and left perfect and has left Loewy length $\alpha+1$ and right Loewy length $\beta+1$. The construction is a modification of an example in [2].

Let $(I, \prec)$ be any partially ordered set. Let $R$ be the set of all $I \times I$ matrices $\left(a_{i, j}\right)$ with coefficients in a field $F$ such that $a_{i, i}=a_{j, j}$ for all $i, j \in I$, and if $i \neq j$ then $a_{i, j}=0$ except for a finite set of pairs $(i, j)$ where $i \prec j$. Then $R$ is a ring under matrix addition and multiplication, and $N=\left\{\left(a_{i, j}\right) \in R \mid a_{i, i}=0\right.$ for all $\left.i \in I\right\}$ is the Jacobson radical of $R$.

Proposition. If $I$ has d.c.c. (respectively a.c.c.), then $R$ is right (left )perfect.

Proof. Since $R / N \approx F$, we need only show that $N$ is right (left) $T$-nilpotent. Let $\left\{r_{n}\right\}$ be a sequence of elements of $N$. Let $s_{n}$ $=r_{n} r_{n-1} \cdots r_{1} r_{0}\left(t_{n}=r_{0} r_{1} \cdots r_{n-1} r_{n}\right)$. Let $F_{n}\left(G_{n}\right)$ be the finite subset of $I$ consisting of all those $i \in I$ such that there exists $k \in I$ with

Received by the editors July $17,1970$.

AMS 1970 subject classifications. Primary 16A22, 16A48; Secondary 16A42.

Key words and phrases. Perfect rings, nil radical, transfinite Loewy length.

1 The author gratefully acknowledges financial support from the NSF under grant GP-19856. 
$\left(s_{n}\right)_{i, k} \neq 0 \quad\left(\left(t_{n}\right)_{k, i} \neq 0\right)$. For $i \in F_{n} \quad\left(G_{n}\right)$ let $f(i)=\left\{j \in F_{n+1} \mid j \prec i\right\}$ $\left(g(i)=\left\{j \in G_{n+1} \mid j \succ i\right\}\right)$. Let $j \in F_{n+1}\left(j^{\prime} \in G_{n+1}\right)$. Since pre- (post-) multiplication by an element of $N$ gives a nonzero product only if some first (second) index is decreased (increased), $j \in f(i)\left(j^{\prime} \in g\left(i^{\prime}\right)\right)$ for some $i \in F_{n}\left(i^{\prime} \in G_{n}\right)$. Consequently every element of $F_{n+1}\left(G_{n+1}\right)$ is connected by a path to an element of $F_{0}\left(G_{0}\right)$. The chain condition on $I$ implies that there are no paths of infinite length in the resulting graph. By the König graph theorem, the lengths of paths starting at $F_{0}\left(G_{0}\right)$ is bounded, say by $m$. But then $F_{m+1}=\varnothing\left(G_{m+1}=\varnothing\right)$, so $s_{m+1}=0\left(t_{m+1}=0\right)$.

We note that the converse of this proposition is also true, as a strictly ascending or descending chain will give rise to a sequence of elements with nonzero finite products.

Now let $\varphi$ and $\psi$ be any infinite ordinals, considered as the set of all their predecessors. Let $I=\varphi \times \psi$, and set $(\alpha, \beta) \prec(\gamma, \delta)$ if and only if $\alpha<\gamma$ and $\beta>\delta$. Then $\preceq$ defines a partial ordering on $I$. Since a descending (ascending) chain in $(I, \preceq)$ gives rise to a descending chain of ordinals in the first (second) coordinate, $I$ has both d.c.c. and a.c.c. Hence the corresponding ring $R$ is both left and right perfect.

For each $r \in N$, let

$$
\begin{aligned}
& A_{r}=\left\{(\alpha, \beta) \in I \mid(r)_{(\alpha, \beta), i} \neq 0 \text { for some } i \in I\right\}, \\
& B_{r}=\left\{\alpha \mid(\alpha, \beta) \in A_{r} \text { for some } \beta \in \psi\right\}, \\
& C_{r}=\left\{\beta \mid(\alpha, \beta) \in A_{r} \text { for some } \alpha \in \varphi\right\} .
\end{aligned}
$$

Let $\mu=\sup \{\alpha \mid \alpha$ a limit ordinal $\leqq \psi\}$. Then $\psi=\mu+n$ for some finite ordinal $n$.

Proposition. If $\left\{N_{\alpha}\right\}$ is Bass' left Loewy series for $R$, then

$$
\begin{aligned}
& N_{\alpha}=\left\{r \in N \mid \inf C_{r} \geqq \mu+(n-\alpha)\right\}+\left\{r \in N \mid B_{r} \subseteq \alpha\right\} \\
& \quad \text { for } 0 \leqq \alpha \leqq n ; \\
& N_{\alpha}=N_{n}+\left\{r \in N \mid B_{r} \subseteq \alpha\right\} \quad \text { for } n \leqq \alpha<\phi .
\end{aligned}
$$

In particular, the left Loewy length of $R$ is $\varphi+1$ if $\varphi$ is a limit ordinal, or $\varphi$ otherwise. By symmetry, the right Loewy length is $\psi+1$ or $\psi$, depending on whether or not $\psi$ is a limit ordinal.

Proof. We use induction on $\alpha$. If $\alpha=0$, the result is immediate. Now assume it holds for all $\beta<\alpha$. If $\alpha$ is a limit ordinal, it follows by taking unions of equals. Hence we are left with the case that $\alpha=\beta+1$ for some ordinal $\beta$. Then $N_{\alpha}=\left\{r \in R \mid N r \subseteq N_{\beta}\right\}$, and it is clear that $N_{\alpha} \subseteq N$ since $N_{\beta} \neq N$. Since pre-multiplication by an element of $N$ 
decreases first components and increases second components, any element in the right-hand side of $\left({ }^{*}\right)$ is sent into $N_{\beta}$ by pre-multiplication by $N$. If $N r \subseteq N_{\beta}$ for some $r \neq 0$, let $(\gamma, \delta) \in A_{r}$. If $\gamma \geqq \alpha$, either $\delta+1=\psi$ or $s r \in N_{\beta}$, where $s$ has zeros everywhere except position $(\beta, \delta+1),(\gamma, \delta)$. Then $(\beta, \delta+1) \in A_{s r}$ so $\delta+1 \geqq \mu+(n-\beta)$. In all cases, either $\gamma<\alpha$ or $\delta \geqq \mu+(n-\beta-1)$, so $r$ is in the right-hand side of $\left({ }^{*}\right)$.

Since any element of $N$ belongs to $N_{\alpha}$ for some $\alpha<\varphi$, the left Loewy length of $R=\varphi+1$ if $N_{\varphi}=N=\mathrm{U}_{\alpha<\varphi} N_{\alpha}$, and it is $\varphi$ if $N=N_{\varphi-1}$.

Since the situation is completely symmetrical, the right Loewy length of $R$ is $\psi+1$ if $\psi$ is a limit ordinal, and $\psi$ otherwise. To get a one-sided perfect ring with arbitrary Loewy length, just use one ordinal for indexing.

\section{BiBLIOGRAPHY}

1. E. Artin, C. J. Nesbitt and R. M. Thrall, Rings with minimum condition, University of Michigan Publ. Math., no. 1, Univ. of Michigan Press, Ann Arbor, Mich., 1944. MR 6, 33.

2. H. Bass, Finitistic dimension and a homological generalization of semi-primary rings, Trans. Amer. Math. Soc. 95 (1960), 466-488. MR 28 \#1212.

Rutgers State University, New Brunswick, New Jersey 08903 